\title{
Further results on Parity Combination Cordial Labeling
}

\author{
Mohamed Seoud ${ }^{1}$ and Mohamed Aboshady ${ }^{2 *}$
}

\author{
* Correspondence: Mohamed. \\ Aboshady@bue.edu.eg \\ ${ }^{2}$ Department of Basic Science, \\ Faculty of Engineering, The British \\ University in Egypt, Cairo, Egypt \\ Full list of author information is \\ available at the end of the article
}

\begin{abstract}
Let $G$ be a $(p, q)$-graph. Let $f$ be an injective mapping from $V(G)$ to $\{1,2, \ldots, p\}$. For each edge $x y$, assign the label $\left(\begin{array}{l}x \\ y\end{array}\right)$ or $\left(\begin{array}{l}y \\ x\end{array}\right)$ according as $x>y$ or $y>x$. Call $f$ a parity combination cordial labeling if $\left|e_{f}(0)-e_{f}(1)\right| \leq 1$, where $e_{f}(0)$ and $e_{f}(1)$ denote the number of edges labeled with an even number and an odd number, respectively. In this paper we make a survey on all graphs of order at most six and find out whether they satisfy a parity combination cordial labeling or not and get an upper bound for the number of edges $q$ of any graph to satisfy this condition and describe the parity combination cordial labeling for two families of graphs.

Math Subject Classification: 05C78, 05C85, 05C30
\end{abstract}

\section{Introduction}

In this paper we will deal with finite simple undirected graphs. By $G(V, E)$ we mean a graph with $p$ vertices and $q$ edges, where $p=|V|$ and $q=|E|$. We follow Harary [1] for standard terminology and notations, and see Gallian [2] for more details on graph labelings.

Definition 1.1 [3] For a graph $G(p, q)$, let $f$ be an injective mapping from $V(G)$ to $\{1$, $2, \ldots, p\}$. For each edge $x y$, assign the label $\left(\begin{array}{l}x \\ y\end{array}\right)$ or $\left(\begin{array}{l}y \\ x\end{array}\right)$ according as $x>y$ or $y>x$. Call $f$ a parity combination cordial labeling if $\left|e_{f}(0)-e_{f}(1)\right| \leq 1$, where $e_{f}(0)$ and $e_{f}(1)$ denote the number of edges labeled with an even number and an odd number, respectively. A graph with a parity combination cordial labeling is called a parity combination cordial graph (PCCG).

Ponraj, Sathish Narayanan, and Ramasamy [3, 4] proved that the following are parity combination cordial graphs: paths, cycles, stars, triangular snakes, alternate triangular snakes, olive trees, combs, crowns, fans, umbrellas, $\boldsymbol{P}_{n}^{2}$, helms, dragons, bistars, butterfly graphs, and graphs obtained from $C_{n}$ and $K_{1, m}$ by unifying a vertex of $C_{n}$ and a pendent vertex of $K_{1, m}$. They also proved that $W_{n}$ admits a parity combination cordial labeling if and only if $n \geq 4$, and conjectured that for $n \geq 4, K_{n}$ is not a parity combination cordial graph. They also proved that if $G$ is a parity combination cordial graph, then $\boldsymbol{G} \cup \boldsymbol{P}_{\boldsymbol{n}}$ is also parity combination cordial if $n \neq 2,4$.

(c) The Author(s). 2020 Open Access This article is licensed under a Creative Commons Attribution 4.0 International License, which permits use, sharing, adaptation, distribution and reproduction in any medium or format, as long as you give appropriate credit to the original author(s) and the source, provide a link to the Creative Commons licence, and indicate if changes were made. The images or other third party material in this article are included in the article's Creative Commons licence, unless indicated otherwise in a credit line to the material. If material is not included in the article's Creative Commons licence and your intended use is not permitted by statutory regulation or exceeds the permitted use, you will need to obtain permission directly from the copyright holder. To view a copy of this licence, visit http://creativecommons.org/licenses/by/4.0/. 
In this paper we try to present some further results, we give the parity combination cordial labeling (PCCL) of all graphs of order at most six, make an algorithm that identify whether any graph of order $p$ and size $q$ can be a PCCG or not, give an upper bound to the number of edges of any graph which satisfy this condition and finally describe the PCCL function of the two graphs $K_{2, n}$ and $P_{n}^{(t)}$.

\section{General results}

Proposition 2.1 For a simple graph $G$ with $q$-edges, if $G$ is PCCL and $q$ is even then $G \pm e$ is PCCG.

Proof Since $G$ is PCCL and $q$ is even, then $e_{f}(0)=e_{f}(1)$. Adding a new edge $e$ will lead to $\left|e_{f}(0)-e_{f}(1)\right|=1$, satisfying the PCCL condition.

Proposition 2.2[2] $\left(\begin{array}{l}n \\ 2\end{array}\right)$ is even if $n \equiv 0,1(\bmod 4)$ and odd if $n \equiv 2,3(\bmod 4)$.

Proposition $2.3\left(\begin{array}{l}n \\ 3\end{array}\right)$ is even if $n \equiv 0,1,2(\bmod 4)$ and odd if $n \equiv 3(\bmod 4)$.

Proof For $n \equiv 1(\bmod 4)$, we have three cases.

Case 1: $n=12 t+1 \Longrightarrow\left(\begin{array}{l}n \\ 3\end{array}\right)=\frac{(12 t+1)(12 t)(12 t-1)}{6}=2 t(12 t+1)(12 t-1) \Rightarrow$ even.

Case 2: $n=12 t+5 \Rightarrow\left(\begin{array}{l}n \\ 3\end{array}\right)=\frac{(12 t+5)(12 t+4)(12 t+3)}{6}=2(12 t+5)(3 t+1)(4 t+1) \Rightarrow$ even.

Case 3: $n=12 t+9 \Rightarrow\left(\begin{array}{l}n \\ 3\end{array}\right)=\frac{(12 t+9)(12 t+8)(12 t+7)}{6}=2(4 t+3)(3 t+2)(12 t+7) \Rightarrow$ even.

Similarly for $n \equiv 0,2(\bmod 4)$.

For $n \equiv 3(\bmod 4)$, we have also three cases.

Case $1: n=12 t+3 \Rightarrow\left(\begin{array}{l}n \\ 3\end{array}\right)=\frac{(12 t+3)(12 t+2)(12 t+1)}{6}=(4 t+1)(6 t+1)(12 t+1) \Rightarrow$ odd.

Case $2: n=12 t+7 \Rightarrow\left(\begin{array}{l}n \\ 3\end{array}\right)=\frac{(12 t+7)(12 t+6)(12 t+5)}{6}=(12 t+7)(2 t+1)(12 t+5) \Rightarrow$ odd.

Case 3: $n=12 t+11 \Longrightarrow\left(\begin{array}{l}n \\ 3\end{array}\right)=\frac{(12 t+11)(12 t+10)(12 t+9)}{6}=(12 t+11)(6 t+5)(4 t+3) \Longrightarrow$ odd.

Proposition 2.4 All simple connected graphs of order at most five are PCCG except $K_{4}, K_{5}$, which is proved later using Algorithms 2.5 and 2.7, as shown in Fig. 1.

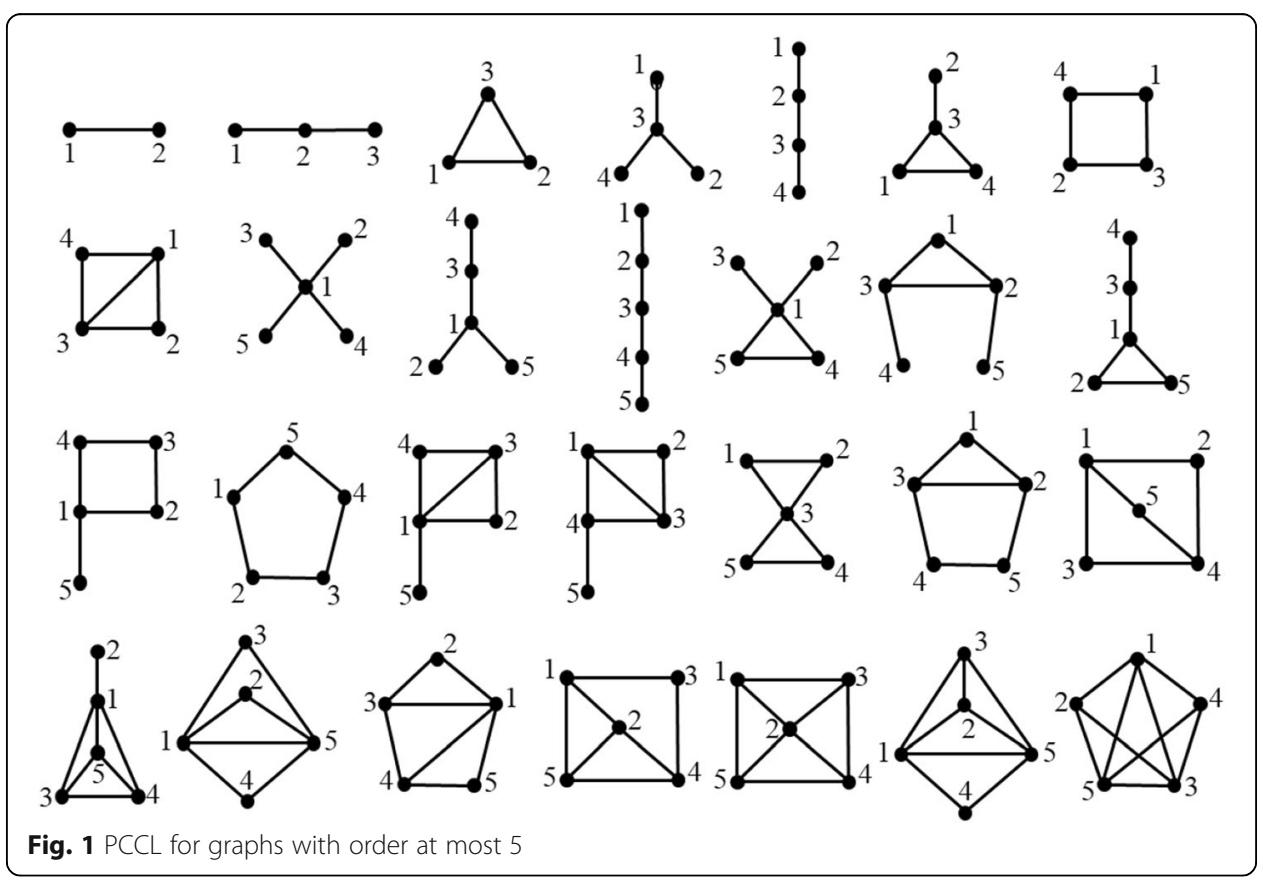


Algorithm 2.5 We made an algorithm to test any graph whether it is PCCG or not.

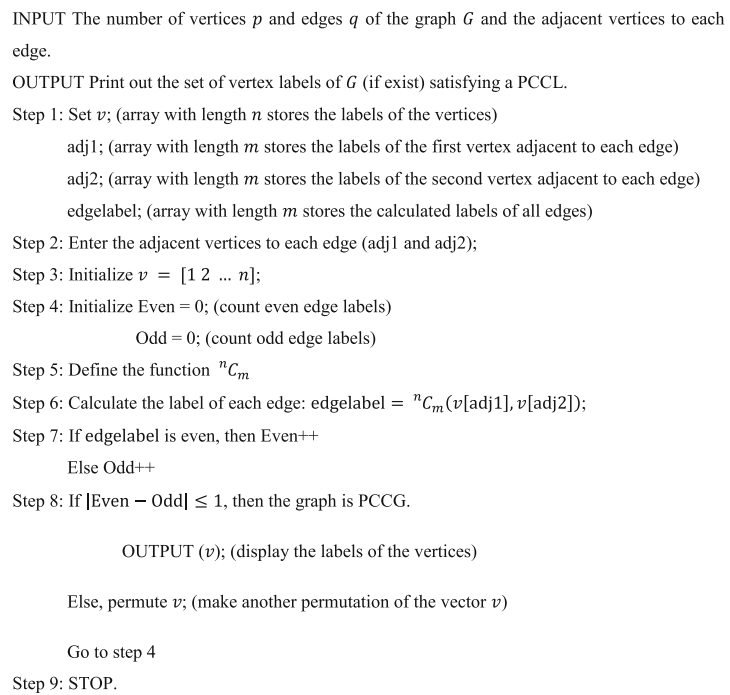

Proposition 2.6 All simple graphs of order six are PCCG, as shown in Appendix 1, except $K_{6}, K_{5} \cup P_{1}, K_{6}-e$ and the following graph (using Algorithm 2.5).

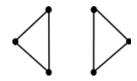

The upper bound for the number of edges $q$ of any graph with $n \leq 100$ vertices to satisfy a PCCL is computed using the following algorithm and shown in Table 1.

Algorithm 2.7 In this algorithm we count the number of even entries and odd entries that are greater than one in the Pascal's triangle and compute twice the smaller number plus one.

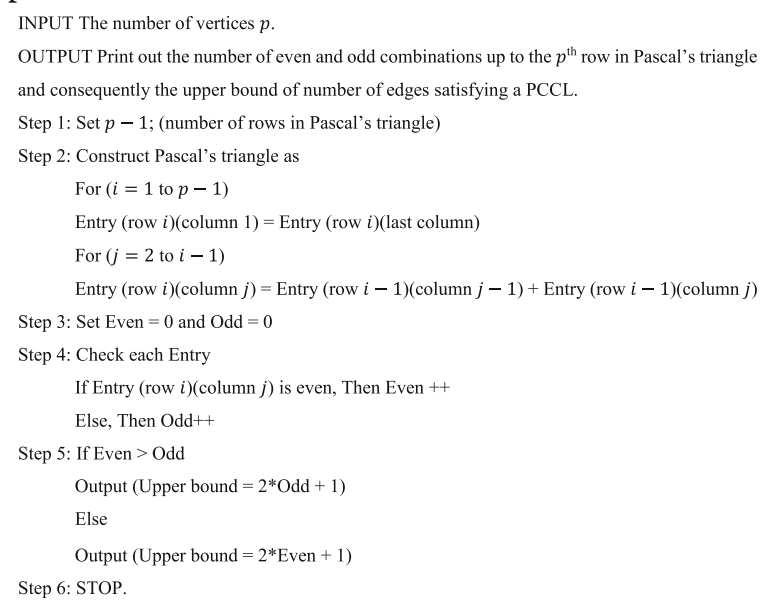

Based on the upper bound listed in Table 1, the following conjecture gives an upper bound for the number of edges of any graph to be PCCG.

Conjecture 2.8 The upper bound for the number of edges of any graph with $n$ vertices $(\forall n \in \mathbb{N}-\{3,7\}$ and $n \geq 2)$ to satisfy a PCCL is given by

$$
1+2 \sum_{i=2}^{n}\left[2^{s b(i)}-2\right]
$$

where $s b(i)$ is number of times the digit 1 occurs in the binary representation of $i$. 
Table 1 The upper bound for the number of edges of a graph with $n$ vertices

\begin{tabular}{|c|c|c|c|c|c|c|c|c|}
\hline$n$ & Upper bound & $\left|K_{n}\right|$ & $n$ & Upper bound & $\left|K_{n}\right|$ & $n$ & Upper bound & $\left|K_{n}\right|$ \\
\hline 2 & 1 & 1 & 35 & 381 & 595 & 68 & 1229 & 2278 \\
\hline 3 & 3 & 3 & 36 & 385 & 630 & 69 & 1241 & 2346 \\
\hline 4 & 5 & 6 & 37 & 397 & 666 & 70 & 1253 & 2415 \\
\hline 5 & 9 & 10 & 38 & 409 & 703 & 71 & 1281 & 2485 \\
\hline 6 & 13 & 15 & 39 & 437 & 741 & 72 & 1285 & 2556 \\
\hline 7 & 19 & 21 & 40 & 441 & 780 & 73 & 1297 & 2628 \\
\hline 8 & 25 & 28 & 41 & 453 & 820 & 74 & 1309 & 2701 \\
\hline 9 & 29 & 36 & 42 & 465 & 861 & 75 & 1337 & 2775 \\
\hline 10 & 33 & 45 & 43 & 493 & 903 & 76 & 1349 & 2850 \\
\hline 11 & 45 & 55 & 44 & 505 & 946 & 77 & 1377 & 2926 \\
\hline 12 & 49 & 66 & 45 & 533 & 990 & 78 & 1405 & 3003 \\
\hline 13 & 61 & 78 & 46 & 561 & 1035 & 79 & 1465 & 3081 \\
\hline 14 & 73 & 91 & 47 & 621 & 1081 & 80 & 1469 & 3160 \\
\hline 15 & 101 & 105 & 48 & 625 & 1128 & 81 & 1481 & 3240 \\
\hline 16 & 101 & 120 & 49 & 637 & 1176 & 82 & 1493 & 3321 \\
\hline 17 & 105 & 136 & 50 & 649 & 1225 & 83 & 1521 & 3403 \\
\hline 18 & 109 & 153 & 51 & 677 & 1275 & 84 & 1533 & 3486 \\
\hline 19 & 121 & 171 & 52 & 689 & 1326 & 85 & 1561 & 3570 \\
\hline 20 & 125 & 190 & 53 & 717 & 1378 & 86 & 1589 & 3655 \\
\hline 21 & 137 & 210 & 54 & 745 & 1431 & 87 & 1649 & 3741 \\
\hline 22 & 149 & 231 & 55 & 805 & 1485 & 88 & 1661 & 3828 \\
\hline 23 & 177 & 253 & 56 & 817 & 1540 & 89 & 1689 & 3916 \\
\hline 24 & 181 & 276 & 57 & 845 & 1596 & 90 & 1717 & 4005 \\
\hline 25 & 193 & 300 & 58 & 873 & 1653 & 91 & 1777 & 4095 \\
\hline 26 & 205 & 325 & 59 & 933 & 1711 & 92 & 1805 & 4186 \\
\hline 27 & 233 & 351 & 60 & 961 & 1770 & 93 & 1865 & 4278 \\
\hline 28 & 245 & 378 & 61 & 1021 & 1830 & 94 & 1925 & 4371 \\
\hline 29 & 273 & 406 & 62 & 1081 & 1891 & 95 & 2049 & 4465 \\
\hline 30 & 301 & 435 & 63 & 1205 & 1953 & 96 & 2053 & 4560 \\
\hline 31 & 361 & 465 & 64 & 1205 & 2016 & 97 & 2065 & 4656 \\
\hline 32 & 361 & 496 & 65 & 1209 & 2080 & 98 & 2077 & 4753 \\
\hline 33 & 365 & 528 & 66 & 1213 & 2145 & 99 & 2105 & 4851 \\
\hline 34 & 369 & 561 & 67 & 1225 & 2211 & 100 & 2117 & 4950 \\
\hline
\end{tabular}

In Table 2 we compare the upper bound calculated from Algorithm 2.7 and that calculated from Conjecture 2.8 for $n=100$ vertices and found that they match in all cases except for $n=3$ and $n=7$.

\section{A PCCL of two graphs}

In this section we present a PCCL of two families of graphs, the graph $K_{2, n}$ and the graph $P_{n}^{(t)}$ which is the one point union of $t$ copies of $P_{n}$.

Proposition 3.1: The graph $K_{2, n}$ is PCCG for $n \equiv 0,2,3(\bmod 4)$. 
Table 2 The upper bound for the number of edges of a graph with $n$ vertices

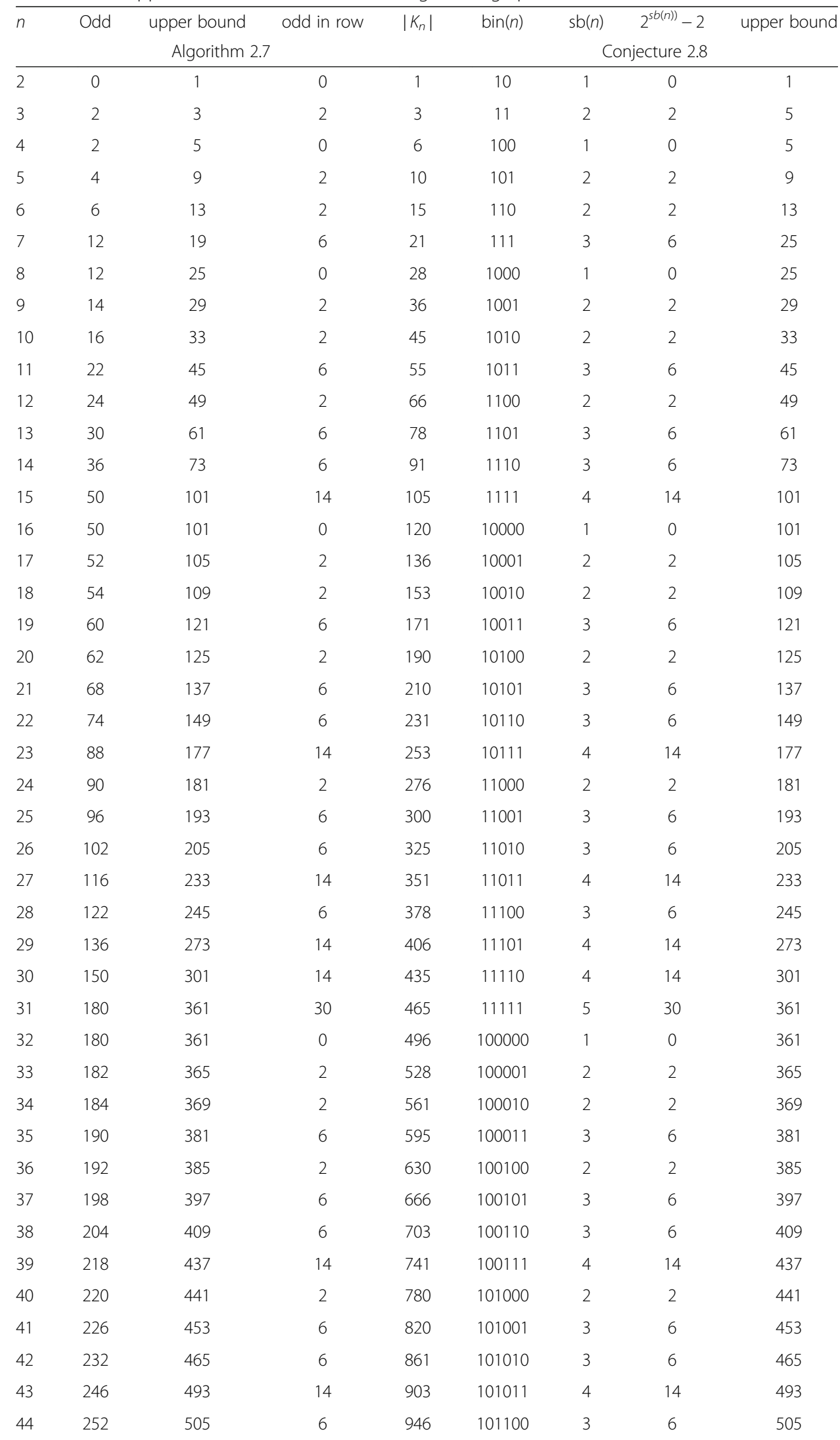


Table 2 The upper bound for the number of edges of a graph with $n$ vertices (Continued)

\begin{tabular}{|c|c|c|c|c|c|c|c|c|}
\hline \multirow[t]{2}{*}{ n } & Odd & upper bound & odd in row & \multirow[t]{2}{*}{$\left|K_{n}\right|$} & $\operatorname{bin}(n)$ & $\mathrm{sb}(n)$ & $2^{s b(n))}-2$ & upper bound \\
\hline & \multicolumn{3}{|c|}{ Algorithm 2.7} & & \multicolumn{4}{|c|}{ Conjecture 2.8} \\
\hline 45 & 266 & 533 & 14 & 990 & 101101 & 4 & 14 & 533 \\
\hline 46 & 280 & 561 & 14 & 1035 & 101110 & 4 & 14 & 561 \\
\hline 47 & 310 & 621 & 30 & 1081 & 101111 & 5 & 30 & 621 \\
\hline 48 & 312 & 625 & 2 & 1128 & 110000 & 2 & 2 & 625 \\
\hline 49 & 318 & 637 & 6 & 1176 & 110001 & 3 & 6 & 637 \\
\hline 50 & 324 & 649 & 6 & 1225 & 110010 & 3 & 6 & 649 \\
\hline 51 & 338 & 677 & 14 & 1275 & 110011 & 4 & 14 & 677 \\
\hline 52 & 344 & 689 & 6 & 1326 & 110100 & 3 & 6 & 689 \\
\hline 53 & 358 & 717 & 14 & 1378 & 110101 & 4 & 14 & 717 \\
\hline 54 & 372 & 745 & 14 & 1431 & 110110 & 4 & 14 & 745 \\
\hline 55 & 402 & 805 & 30 & 1485 & 110111 & 5 & 30 & 805 \\
\hline 56 & 408 & 817 & 6 & 1540 & 111000 & 3 & 6 & 817 \\
\hline 57 & 422 & 845 & 14 & 1596 & 111001 & 4 & 14 & 845 \\
\hline 58 & 436 & 873 & 14 & 1653 & 111010 & 4 & 14 & 873 \\
\hline 59 & 466 & 933 & 30 & 1711 & 111011 & 5 & 30 & 933 \\
\hline 60 & 480 & 961 & 14 & 1770 & 111100 & 4 & 14 & 961 \\
\hline 61 & 510 & 1021 & 30 & 1830 & 111101 & 5 & 30 & 1021 \\
\hline 62 & 540 & 1081 & 30 & 1891 & 111110 & 5 & 30 & 1081 \\
\hline 63 & 602 & 1205 & 62 & 1953 & 111111 & 6 & 62 & 1205 \\
\hline 64 & 602 & 1205 & 0 & 2016 & 1000000 & 1 & 0 & 1205 \\
\hline 65 & 604 & 1209 & 2 & 2080 & 1000001 & 2 & 2 & 1209 \\
\hline 66 & 606 & 1213 & 2 & 2145 & 1000010 & 2 & 2 & 1213 \\
\hline 67 & 612 & 1225 & 6 & 2211 & 1000011 & 3 & 6 & 1225 \\
\hline 68 & 614 & 1229 & 2 & 2278 & 1000100 & 2 & 2 & 1229 \\
\hline 69 & 620 & 1241 & 6 & 2346 & 1000101 & 3 & 6 & 1241 \\
\hline 70 & 626 & 1253 & 6 & 2415 & 1000110 & 3 & 6 & 1253 \\
\hline 71 & 640 & 1281 & 14 & 2485 & 1000111 & 4 & 14 & 1281 \\
\hline 72 & 642 & 1285 & 2 & 2556 & 1001000 & 2 & 2 & 1285 \\
\hline 73 & 648 & 1297 & 6 & 2628 & 1001001 & 3 & 6 & 1297 \\
\hline 74 & 654 & 1309 & 6 & 2701 & 1001010 & 3 & 6 & 1309 \\
\hline 75 & 668 & 1337 & 14 & 2775 & 1001011 & 4 & 14 & 1337 \\
\hline 76 & 674 & 1349 & 6 & 2850 & 1001100 & 3 & 6 & 1349 \\
\hline 77 & 688 & 1377 & 14 & 2926 & 1001101 & 4 & 14 & 1377 \\
\hline 78 & 702 & 1405 & 14 & 3003 & 1001110 & 4 & 14 & 1405 \\
\hline 79 & 732 & 1465 & 30 & 3081 & 1001111 & 5 & 30 & 1465 \\
\hline 80 & 734 & 1469 & 2 & 3160 & 1010000 & 2 & 2 & 1469 \\
\hline 81 & 740 & 1481 & 6 & 3240 & 1010001 & 3 & 6 & 1481 \\
\hline 82 & 746 & 1493 & 6 & 3321 & 1010010 & 3 & 6 & 1493 \\
\hline 83 & 760 & 1521 & 14 & 3403 & 1010011 & 4 & 14 & 1521 \\
\hline 84 & 766 & 1533 & 6 & 3486 & 1010100 & 3 & 6 & 1533 \\
\hline 85 & 780 & 1561 & 14 & 3570 & 1010101 & 4 & 14 & 1561 \\
\hline 86 & 794 & 1589 & 14 & 3655 & 1010110 & 4 & 14 & 1589 \\
\hline 87 & 824 & 1649 & 30 & 3741 & 1010111 & 5 & 30 & 1649 \\
\hline
\end{tabular}


Table 2 The upper bound for the number of edges of a graph with $n$ vertices (Continued)

\begin{tabular}{lcccccccc}
\hline$n$ & Odd & $\begin{array}{c}\text { upper bound } \\
\text { Algorithm } 2.7\end{array}$ & odd in row & $\left|K_{n}\right|$ & bin $(n)$ & $\begin{array}{c}\text { sb }(n) \\
\text { Conjecture 2.8 }\end{array}$ & $2^{\text {sb(n)) }}-2$ & upper bound \\
\hline 88 & 830 & 1661 & 6 & 3828 & 1011000 & 3 & 6 & 1661 \\
89 & 844 & 1689 & 14 & 3916 & 1011001 & 4 & 14 & 1689 \\
90 & 858 & 1717 & 14 & 4005 & 1011010 & 4 & 14 & 1717 \\
91 & 888 & 1777 & 30 & 4095 & 1011011 & 5 & 30 & 1777 \\
92 & 902 & 1805 & 14 & 4186 & 1011100 & 4 & 14 & 1805 \\
93 & 932 & 1865 & 30 & 4278 & 1011101 & 5 & 30 & 1865 \\
94 & 962 & 1925 & 30 & 4371 & 1011110 & 5 & 30 & 1925 \\
95 & 1024 & 2049 & 62 & 4465 & 1011111 & 6 & 62 & 2049 \\
96 & 1026 & 2053 & 2 & 4560 & 1100000 & 2 & 2 & 2053 \\
97 & 1032 & 2065 & 6 & 4656 & 1100001 & 3 & 6 & 2065 \\
98 & 1038 & 2077 & 6 & 4753 & 1100010 & 3 & 6 & 2077 \\
99 & 1052 & 2105 & 14 & 4851 & 1100011 & 4 & 14 & 2105 \\
100 & 1058 & 2117 & 6 & 4950 & 1100100 & 3 & 6 & 2117 \\
\hline & & & & & & & &
\end{tabular}

Proof. Let the set of vertices be $V\left(K_{2, n}\right)=\left\{u_{1}, u_{2} ; v_{1}, v_{2}, \ldots, v_{n}\right\}$ and the set of edges be $E\left(K_{2}\right.$, $\left.{ }_{n}\right)=\left\{u_{1} v_{1}, u_{1} v_{2}, \ldots, u_{1} v_{n} ; u_{2} v_{1}, u_{2} v_{2}, \ldots, u_{2} v_{n}\right\}$. It's clear that $\left|V\left(K_{2, n}\right)\right|=n+2$ and $\left|E\left(K_{2, n}\right)\right|=2 n$. Then for $n \equiv 0,2,3(\bmod 4)$, define the labeling function $f: V\left(K_{2, n}\right) \rightarrow\{1,2, \ldots, p=n+2\}$ by

$$
f\left(u_{1}\right)=1, f\left(u_{2}\right)=2,
$$

and

$$
f\left(v_{i}\right)=i+2,1 \leq i \leq n
$$

Which will lead to $e_{f}(0)=e_{f}(1)=n$ depending on proposition 2.2.

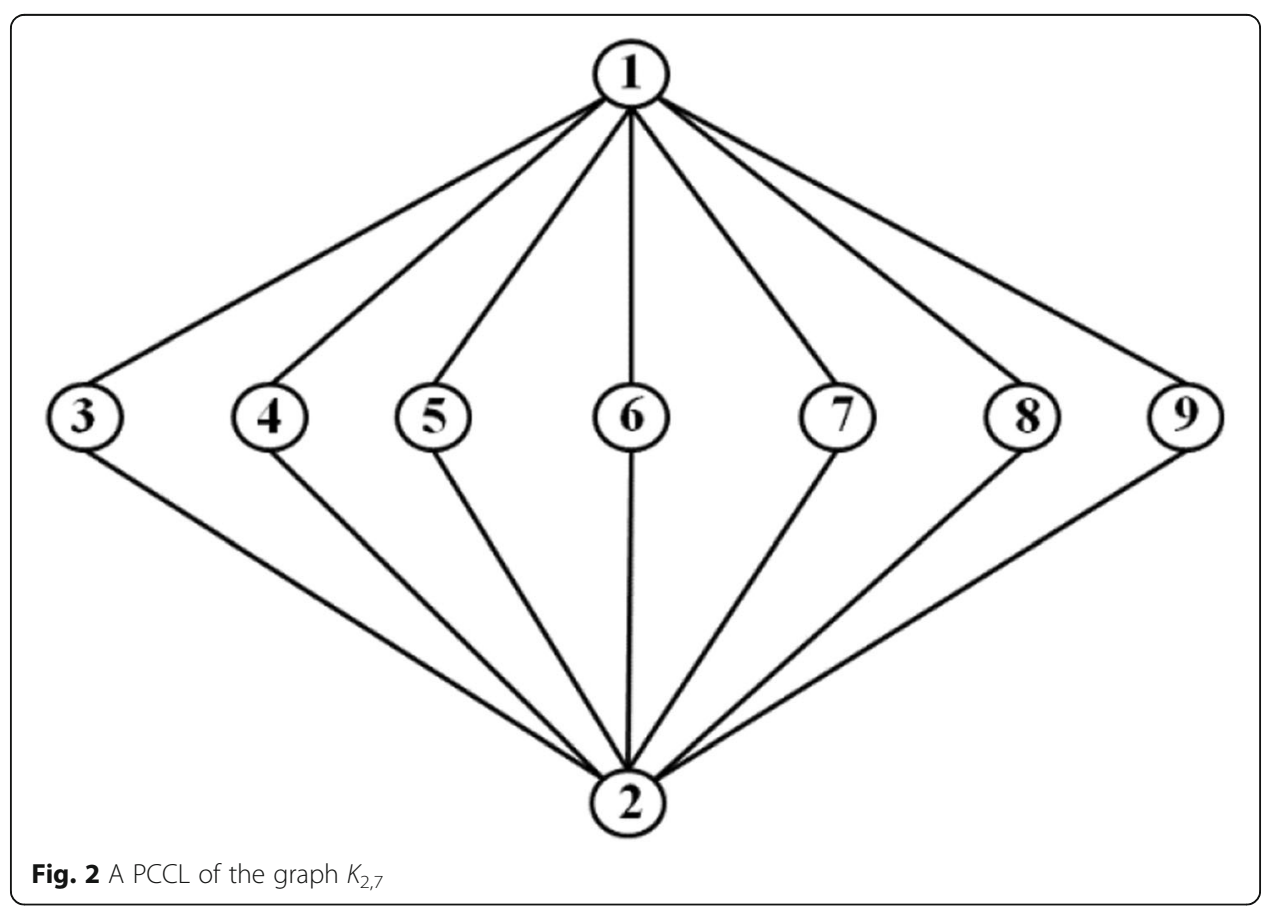


Example: The graph $K_{2,7}$ is PCCG as shown in Fig. 2.

Proposition 3.2: The graph $P_{n}^{(t)}$, the one point union of $t$ copies of $P_{n}$, is PCCG.

Proof. Let the set of vertices be $V\left(P_{n}^{(t)}\right)=\left\{v_{1}^{(1)}, v_{2}^{(1)}, \ldots, v_{n}^{(1)} ; v_{2}^{(2)}, \ldots, v_{n}^{(2)} ; \ldots ; v_{2}^{(t)}, \ldots\right.$, $\left.v_{n}^{(t)}\right\}$. It's clear that $\left|V\left(P_{n}^{(t)}\right)\right|=(n-1) t+1$ and $\left|E\left(P_{n}^{(t)}\right)\right|=(n-1) t$. Then define the labeling function $f: V\left(P_{n}^{(t)}\right) \rightarrow\{1,2, \ldots,(n-1) t+1\}$ by

$$
f\left(v_{i}^{(j)}\right)=\left\{\begin{array}{cc}
1, & \text { if } i=j=1 \\
(j-1)(n-1)+i, & 2 \leq i \leq n, \quad 1 \leq j \leq t
\end{array}\right.
$$

This function will lead to $0 \leq\left|e_{f}(0)-e_{f}(1)\right| \leq 1$ depending on proposition 2.2. Example: The graph $P_{7}^{(4)}$ is PCCG as shown in Fig. 3.



Fig. 3 A PCCL of the graph $P_{7}^{(4)}$ 


\section{Appendix}

Appendix 1: All graphs of order six according to Harary [2] that satisfy a PCCL

$$
\begin{aligned}
& 2^{2}=0^{2}
\end{aligned}
$$

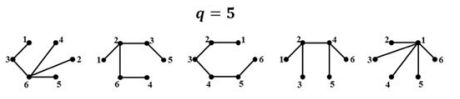



$$
\begin{aligned}
& q=6 \\
& \text { * } \square \cdot \square \cdot \square \\
& \text { W } \\
& \text { W } \\
& q=7
\end{aligned}
$$

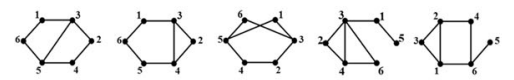

$$
\begin{aligned}
& \mathbb{N}=\mathbb{N}
\end{aligned}
$$

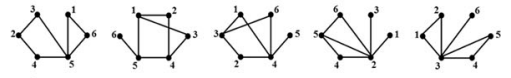

$$
\begin{aligned}
& \mathbb{V}^{2} \cdot \sqrt{1} \cdot \sqrt{1} \\
& \$ 2 \mathbb{N} \\
& \Leftrightarrow \Delta \\
& \Delta \cdot \Delta \cdot N \quad \& D \\
& \forall \cdot \square \cdot \Delta \cdot \\
& \sum_{1} \mathbb{X} \\
& q=9 \\
& \rightleftarrows \Leftrightarrow \\
& \text { \#. } \Delta, \nabla \otimes \\
& \mathbb{A} \otimes \cdot \mathbb{\Delta} \otimes
\end{aligned}
$$

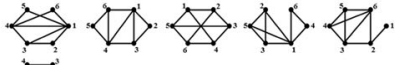

$$
\begin{aligned}
& \text { D } \\
& \Delta \otimes
\end{aligned}
$$

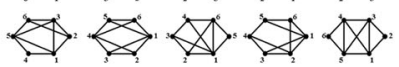

$$
\begin{aligned}
& \leftrightarrow \otimes \\
& q=11
\end{aligned}
$$

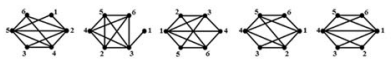

$$
\begin{aligned}
& \notin
\end{aligned}
$$

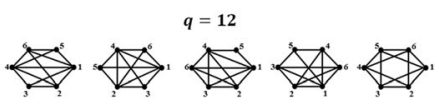

$$
\begin{aligned}
& q=13
\end{aligned}
$$


Acknowledgments

Not applicable.

\section{Authors' contributions}

All authors contributed equally to this manuscript. All authors read and approved the final manuscript.

\section{Funding}

The authors received no financial support for the research, authorship, and/or publication of this article.

\section{Availability of data and materials}

All the data in the manuscript are public.

\section{Competing interests}

The authors declare that they have no competing interests.

\section{Author details}

'Department of Mathematics, Faculty of Science, Ain Shams University, Cairo, Egypt. ${ }^{2}$ Department of Basic Science, Faculty of Engineering, The British University in Egypt, Cairo, Egypt.

Received: 29 November 2019 Accepted: 13 April 2020

Published online: 24 May 2020

\section{References}

1. F. Harary: Graph Theory. Addison-Wesley, Reading, MA (1969). https://shyam.nitk.ac.in/Books/GT\%20Books/Harary.pdf

2. J.A. Gallian: A Dynamic Survey of Graph Labeling. The Elec. J. of Comb.. 21th Ed. (2018). https://www.combinatorics.org/ ojs/index.php/eljc/article/view/DS6/pdf

3. R. Ponraj, S. Sathish Narayanan and A.M.S. Ramasamy: Parity Combination Cordial Labeling of Graphs, Jordan J. of Math. and Stat.. 8(4), (2015) 293-308. https://www.researchgate.net/publication/299031186_Parity_combination_cordial_ labeling_of_graphs

4. R. Ponraj, Rajpal Singh and S. Sathish Narayanan: On parity combination cordial graphs, Palestine Journal of Mathematics. 6(1), (2017) 211-218. http://pjm.ppu.edu/sites/default/files/papers/PJM_MAR_2017_25.pdf

\section{Publisher's Note}

Springer Nature remains neutral with regard to jurisdictional claims in published maps and institutional affiliations.

\section{Submit your manuscript to a SpringerOpen ${ }^{\circ}$ journal and benefit from:}

- Convenient online submission

$\checkmark$ Rigorous peer review

- Open access: articles freely available online

- High visibility within the field

- Retaining the copyright to your article 